\title{
Generating Low-Carbon Heat from Biomass: Life Cycle Assessment of Bioenergy Scenarios
}

\begin{tabular}{|c|c|}
\hline Author: & Andrew Welfle*, Paul Gilbert*, Patricia Thornley*, Anna Stephenson** \\
\hline & $\begin{array}{c}\text { *Tyndall Centre for Climate Change Research, School of Mechanical Aerospace \& Civil } \\
\text { Engineering, University of Manchester, Pariser Building, Sackville Street, Manchester, } \\
\text { M13 9PL }\end{array}$ \\
$\begin{array}{c}\text { **UK Department for Business, Energy \& Industrial Strategy, 3 Whitehall Place, London, } \\
\text { SW1A 2AW }\end{array}$ \\
$\begin{array}{c}\text { Key } \\
\text { Contact: }\end{array}$ & andrew.welfle@manchester.ac.uk \\
\hline
\end{tabular}

\section{Keywords}

Bioenergy, GHG, Biomass, LCA, Residue, Waste, Scenario, Counterfactual

\begin{abstract}
Bioenergy systems will play a key role in many countries achieving their climate change, emission reduction and renewable energy contribution targets. It is important that implemented bioenergy pathways maximise GHG reductions, particularly since demand and competition for biomass resource is likely to increase in future. This research analyses the actual GHG performance of utilising different biomass resources to generate heat. Life cycle assessment (LCA) is undertaken to evaluate 2,092 variants of bioheat options focused on utilising: agricultural and food wastes through anaerobic digestion pathways; straw agricultural residues and UK grown energy crops through combustion pathways. The results show a very broad range of GHG performances. Many pathways demonstrate GHG savings compared to conventional generation, although some have potential to actually increase GHG emissions, rather than reduce them. Variations in GHG performance do not correlate with feedstocks or technologies, but are most sensitive to the inclusion of specific processing steps and the displacement of certain counterfactuals. This suggests that policies should be developed that target resource with high GHG intensity counterfactuals, and where possible avoid energy intensive processing steps such as pelletisation.
\end{abstract}




\section{1) Introduction}

European Governments have greenhouse gas (GHG) emission and renewable energy targets that are bound by the baseline requirements of the Kyoto Protocol (United Nations, 1998), and the European Commission's Renewable Energy Directive (European Commission, 2009). In addition, the UK is legally bound by the 2008 Climate Change Act (UK Government, 2008), to achieve a mandatory 80\% cut in the UK's carbon emissions below 1990 levels by 2050, and a benchmark target to reduce carbon emissions by 35\% below 1990 levels by 2020 (DECC, 2009). In the context of these targets, the UK's Renewable Energy Roadmap (DECC, 2013) confirms the high likelihood that bioenergy systems will contribute an increasingly important role in the UK achieving its climate change, emission reduction and renewable energy contribution targets. As bioenergy pathways are being assumed in many national energy strategies globally, critical assessment of biomass resources and bioenergy processes is essential. A key justification for bioenergy systems is their ability to deliver energy with reduced GHG emissions compared to fossil fuel systems (Nguyen et al., 2010). Therefore a fundamental requirement for bioenergy has to be that any biomass resources utilised, and any activities and processes applied to generate bioenergy has to results in genuine reductions in GHG emissions over the whole process life cycles.

The variability of GHG performance of bioenergy pathways has been highlighted by reports such as that by the UK's SUPERGEN Bioenergy Hub (Adams et al., 2013), where it was confirmed that: many bioenergy pathways can deliver energy with GHG savings compared to fossil fuels; although the specific life cycle processes and activities inherent to the bioenergy pathway and the assumed counterfactual (what would have happened to the land/resource if not used for bioenergy) can be highly influential in determining the overall GHG performance of a bioenergy pathway, and some pathways can have GHG emissions greater than fossil fuels.

Evaluating the overall GHG performance of bioenergy pathways can be challenging and is often disputed due to variations in scope of systems, data inputs or choice of methodologies implemented (Haberl et al., 2012). Bioenergy pathways differ in scope and boundaries and as a consequence, equal levels of variation should be expected when comparing the GHG performance of bioenergy pathways. It should therefore not be assumed that a broad range of feedstocks, conversion processes and end user demands bound together by the concept "bioenergy" should always deliver consistent GHG reductions compared to alternative fossil fuel pathways. Real reductions in GHG emissions are possible from bioenergy, but caution should be applied to ensure that chosen bioenergy pathways deliver genuine GHG reductions (Adams et al., 2013). A life cycle assessment (LCA) approach is the most frequently applied methodology for evaluating the GHG performance of bioenergy pathways (Bowyer et al., 2012), for example as used by Röder et al., 2015 and Thornley et al., 2015. The principal of LCA analysis is to assess the full impact of a pathway - although many of the challenges associated with bioenergy systems may be attributed to the fact that bioenergy is not a single process, but a complex supply chain with extensive physical and socio-economic interfaces that all influence their surroundings - each potential variation within a bioenergy pathway effectively equates to assessing a slightly different "LCA question" (Thornley, 2016).

The EU has developed a series of non-legally binding bioenergy sustainability criteria (European Commission, 2010), that in part set guidelines for the levels of GHG savings that should be achieved through the generation of bioenergy from a given biomass resource in comparison to that from conventional fossil fuel energy. There is 
also a proposed framework for these guideline sustainability criteria to be made progressively more stringent (Panoutsou et al., 2010). Although there are growing concerns about that the scope of methodologies applied for benchmarking the sustainability credentials of different biomass resources, supply chains and bioenergy conversion pathways (Upham and Tomei, 2010). For example the criteria predominantly focuses on the production of biofuels, rather than solid biomass fuels; there is no accounting for changes in land carbon stock unless there is a change in land use; and measuring the GHG impact of biomass for energy relating to indirect land use change (ILUC) is highly uncertain and difficult to model (Ahlgren and Di Lucia, 2014). Therefore the sustainability credentials and potential GHG impact of utilising increasing levels of biomass resources, has become a vital area of discussion with growing interest from Governments and energy and environmental stakeholders.

The UK Department of Energy \& Climate Change (DECC) developed the 'Bioenergy Emissions and Counterfactual' (BEAC) model to provide a scientific tool for investigating the GHG impact of different biomass supply chains, and to evaluate the resulting GHG intensity of generated bioenergy (DECC, 2014a; MacKay and Stephenson, 2014). This research was undertaken in response to the growing consensus that the GHG balance of bioenergy systems can be highly variable and with the UK increasingly targeting bioenergy, there is great reliance on the notion that the UK's bioenergy sector will deliver GHG savings in comparison to fossil fuel generation. DECC's BEAC research focused on evaluating the GHG performance of biomass resources sourced from North America that would potentially be transported to the UK to generate electricity (MacKay and Stephenson, 2014).

This paper presents research where a series of UK biomass resource scenarios were developed with the primary aim of analysing the GHG performance of generating heat bioenergy from UK biomass resources. The research reflects work carried out by the authors working closely with DECC to apply their BEAC analysis methodology to evaluate the GHG performance of generating heat through various bioenergy pathways using key categories of UK biomass resources. An LCA approach is used to evaluate the GHG performance of 2,092 variants of bioheat scenarios utilising UK biomass: agricultural wastes (animal slurries); food wastes; agricultural residues (straws); and purpose grown energy crops - categories of biomass resource identified as representing great potential for the future UK bioenergy sector (Welfle, 2014; Welfle et al., 2014a, 2014b). The generation of heat bioenergy is the focus of the research, as previous work has undertaken similar analysis evaluating the GHG performance of biomass supply chains for power bioenergy pathways (MacKay and Stephenson, 2014). Also, the demands of the UK bio-power sector to 2020 are projected to exceed the UK's domestic supply and increasingly become reliant on imported resources (Welfle, 2014).

\section{2) Methodology}

This research focuses on analysing the GHG performance of different UK biomass resources to generate heat bioenergy, and to investigate the different influences that lead to variability in GHG performance across different bioenergy scenarios.

\subsection{Methodology Framework: Goal and Scope Definition}

A series of unique biomass sourcing and bioenergy generation scenarios were developed with DECC to reflect different potential pathways for generating heat bioenergy from UK biomass. These were modelled through 
developing a spreadsheet analysis tool. Each of these 'bioenergy scenarios' were designed with varying life cycle pathways with different activities and processes inherent to each scenario. A further series of 'counterfactual scenarios' were also developed to allow the analysis of potential GHG impacts or savings that may be achieved through utilising the land/resources for bioenergy generation, rather than the life cycle pathway of the counterfactual.

Through evaluating the GHG impact and energy demand for each life cycle step within both the counterfactual and bioenergy scenarios, the overall GHG emission balance for each bioenergy pathway can be calculated. Figure 1 provides an overview of the methodology framework and the calculation steps applied for determining the overall GHG balance of different biomass resource scenarios.

Figure 1: Overview of the Methodology Calculation Steps for Determining the Overall GHG Impact of Bioenergy Pathways

Figure 2 presents a high level schematic of the calculation pathways, themes and analysis boundaries applicable to the research's bioenergy and counterfactual scenarios. Table 1 summarises the specific processes and activities analysed across the different scenarios. The specific processes and activities evaluated for each category of biomass resources were included following discussions with stakeholders and review of widespread peer-reviewed literature.

Overall GHG performance calculations were undertaken reflecting all combinations of lifecycle activities and processes applicable to each category of biomass resource. The applied calculation assumptions when modelling each life cycle process or activities are listed along with all applicable references within this paper's extensive Supplementary Material.

This analysis methodology represents an attributional form of life cycle assessment (ALCA), where the results generated for each bioenergy pathways are reflective of the specific characteristics of the different bioenergy and counterfactual scenarios. This includes consideration of changes in biogenic carbon across the scenarios to allow a complete accounting of the carbon inventory in accordance to the principles of LCA. Processes with potential GHG impacts outside the different scenario's analysis boundaries are not analysed and are therefore outside the scope of the research.

The key analysis outputs for each of the UK resource bioenergy pathways are: heat bioenergy potential of the different pathways (MWh); the potential GHG performance of generated bioenergy ( $\left.\mathrm{kg} \mathrm{CO}_{2}{ }^{\text {eqv. }} / \mathrm{MWh}\right)$; and a breakdown of the GHG emissions attributed to each step within the bioenergy/counterfactual life cycles of the pathways ( $\mathrm{kg} \mathrm{CO}_{2}{ }^{\text {eqv. }} /$ unit of feedstock).

Figure 2: Life Cycle Themes and Analysis Boundaries of the Research's Bioenergy \& Counterfactual Scenarios for each Category of Biomass Resource

Table 1: Life Cycle Processes and Activities Analysed within the Research's UK Biomass Resource \& Bioenergy Scenarios

\subsection{Research \& Analysis Themes}

The results for each pathway are presented in detail in the Supplementary Material. The analysis in this paper focuses on evaluating the potential for generating heat bioenergy from UK biomass with lower GHG intensities compared to fossil fuel generation; evaluating whether there is variation in bioheat GHG intensities across different bioenergy scenarios; and discussing the influences and impact of any variation in GHG intensities. 


\subsubsection{GHG Performance of UK Biomass Heat Bioenergy Scenarios Compared to Fossil Fuel Generation}

This comparison is undertaken by analysing the range of GHG performance values for the scenarios, and evaluating the mean and median averages values to determine the characteristics of the results. Graphs are also presented to provide further visualisation of the results of selected scenarios. These are compared against the equivalent UK GHG performance value for generating heat from natural gas $\left(261.5 \mathrm{kgCO}_{2}{ }^{\text {eqv }} / \mathrm{MWh}^{\mathrm{a}}\right.$ heat (MacKay and Stephenson, 2014)) and against the UK Government's sustainability target for heat bioenergy generation (125.3 $\mathrm{kgCO}_{2}{ }^{\text {eqv. }} / \mathrm{MWh}$ heat (DECC, 2014b)).

\subsubsection{Evaluating the Influences of Variation in GHG Performance Across the Scenarios}

An assessment of the influencing factors that determine the GHG performance of a given scenario is undertaken by analysing the different levels of GHG emissions attributed to the activities and process specific to different scenarios. By providing a breakdown of the overall emissions within each scenario, it is possible to assess the GHG impact of specific practices within the biomass resource supply chains and that of specific bioenergy conversion steps - therefore highlighting opportunities for developing best practice bioenergy pathways that avoid high GHG impact methods. Likewise this analysis may also allow high GHG impact pathways within the counterfactual scenarios to be identified - highlighting specific types of resource to be targeted for mobilisation/production for bioenergy that will result in emissions savings through mitigating counterfactual processes and activities.

\section{3) Results}

\subsection{The GHG Performance of Generating Heat from Categories of UK Biomass}

The following section presents the GHG performance values for the generation of heat bioenergy from different categories of UK biomass. These are compared against the equivalent value for generating heat from natural gas in the UK, and the UK's RHI sustainability target for heat bioenergy generation.

Table 2 presents the range (minimum to maximum) and the average (mean and median) GHG performance values for generating heat from each category of UK biomass resource. The range and average values are reflective of all the analysed scenarios for each type of biomass - all combinations of biomass resource supply chain pathways, bioenergy conversion pathways and counterfactual pathways applicable to each category of biomass (listed in Table 1).

Each of the GHG performance values presented within Table 2 are categorised through shading. GHG performance values have been darkly shaded to reflect the generation of heat with greater GHG intensity compared to generation from natural gas in the UK. GHG performance values that have been lightly shaded reflect the generation of heat with GHG intensity lower than that by natural gas, but exceeding the UK's sustainability target for heat bioenergy generation. GHG performance values with no shading reflect the generation of heat with GHG intensity below the UK's sustainability target.

Table 2: UK Heat Bioenergy GHG Performance Results from across the Research Scenarios

The minimum GHG performance values presented in Table 2 provide an indication of whether it is possible to generate heat with GHG intensities within the UK's target sustainability threshold from the categories of UK

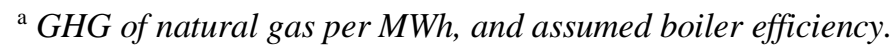


biomass analysed. It shows that it is possible to generate 'sustainable heat' from all the categories of UK biomass analysed. However the minimum values also highlight that within the energy crop scenarios where the applied counterfactual assumes that 'the land would otherwise have been abandoned and left to reverted to a natural (forest) system', the GHG performance of heat generated would exceed the UK's sustainability target (apart from the willow chip scenario), but fall below the equivalent GHG performance of UK natural gas generation.

The maximum GHG performance values presented in Table 2 provide an indication of where heat generated from the different categories of UK biomass exceed the UK's sustainability target and/or the equivalent value for natural gas generation. Table 2 highlights that the maximum heat GHG intensities from all the analysed straw scenarios and the food waste anaerobic digestion scenarios fall within UK's threshold for sustainable heat. In contrast, the results highlight that there is potential for heat generated from agricultural waste (slurry) anaerobic digestion pathways to exceed the equivalent GHG performance values for natural gas - although the GHG performance values are improved when slurry is co-digested with either food waste or energy crops (maize).

The maximum heat GHG intensity values for the energy crop scenarios that utilised chip feedstock are shown to reflect the most favourable GHG performances - depending on the assumed counterfactual, these scenarios are shown to generate heat within the UK's sustainability target threshold. The maximum values highlight that there is potential for all the analysed energy crop scenarios that utilise pellet feedstocks to exceed UK's sustainability target threshold. Where the applied energy crop counterfactual assumes that the land would otherwise have been abandoned and left to reverted to natural (forest) system', heat GHG intensity values may exceed those of equivalent natural gas generation.

Overall the GHG performance values presented within Table 2 document that there is favourable tendency that heat generated within scenarios reflective of either the agricultural and food waste scenarios, straw residue scenarios and energy crop chip feedstock scenarios would generate heat with GHG intensities below the UK target sustainability threshold. In contrast, the average GHG performance values within Table 2 also highlight that there is greater tendency for heat generated within pathways reflective the energy crop pellet scenarios to have GHG intensities greater than the UK sustainability threshold.

\subsection{Evaluating Variability GHG Performance across the Bioenergy Scenarios}

The following section presents a series of figures that are designed to highlight the variability in GHG performance when generating heat bioenergy from different categories of UK biomass, and to allow assessment of the factors influencing the variability.

Variability in GHG performance is demonstrated by the presentation of a series of specific heat GHG intensity values - each reflecting the generation of heat from the same categories of biomass, albeit with different resource supply chains and conversion pathways. Figure 3 supported by the key in Table 3 presents the GHG performance values of specific food waste and cattle slurry scenarios, where heat is generated by anaerobic digestion and biogas combustion pathways. Figure 4 supported by the key in Table 4 presents the GHG performance values of specific willow short rotation coppice (SRC) scenarios where heat is generated by combustion pathways. 
The analysis presented for the willow energy crop scenarios in Figure 4 provides a snapshot of overall GHG performance assuming that the land has been either continually producing energy crops or following an alternative counterfactual over a 40 year timescale. This allows an assessment of GHG performance reflective of several crop cultivation cycles and allows time for assessment of carbon fluxes associated with the counterfactual land use pathways.

\subsubsection{GHG Performance of Generating Bioheat from Food \& Agricultural Waste Resources}

\section{Figure 3: GHG Performance of Selected UK Food Waste \& Cattle Slurry Anaerobic Digestion Scenarios}

Table 3: Key to Analyses Presented in Figure 3

Two immediate conclusions can be drawn through evaluating the GHG intensity results presented in Figure 3: that generating heat bioenergy from UK food wastes and cattle slurries within AD pathways can result in potentially large GHG savings for emission inventories; but at the same time there is potentially large variation in GHG performance depending on the specific characteristic of the resources supply chain and chosen bioenergy conversion pathway.

Figure 3 shows that heat bioenergy generated from food wastes through AD pathways reflective of the analysed scenarios, will result in bioenergy with GHG intensities below the UK's target sustainability threshold. Scenarios where the food wastes would otherwise have been sent to landfill are shown to generate bioenergy with potentially large net negative GHG intensities over the resource's life cycle and bioenergy pathway. Whereas food wastes that would otherwise have been composted are shown to potentially generate bioenergy with marginally higher GHG intensities, albeit well below the UK's target sustainability threshold.

The GHG intensity of heat bioenergy generated from $\mathrm{AD}$ pathways reflective of the analysed scenarios using cattle slurry, or cattle slurry with food/energy crop co-digestate feedstocks, also largely falls below the UK's target sustainability threshold. There is a clear trend that where the counterfactual involves good practices and GHG emission reduction techniques (e.g. the use of closed tank waste storage systems), the overall GHG emissions associated with a bioenergy scenario increases. On the other hand, scenarios involving counterfactuals with high GHG impact processes are shown to reflect large net negative GHG intensities. For example, the scenarios where lagoon storage systems are mitigated in the counterfactual are shown to result in bioenergy pathways with lowest bioenergy GHG intensities. Scenarios where open tank slurry storage systems are mitigated in the counterfactual also display potential GHG intensities far below the UK's target sustainability threshold.

Another trend evident in the presented results is that where wastes would otherwise have been managed through broadcast spreading on the land within the counterfactual (a high GHG impact practice), the scenario's overall GHG intensities are lower as a result of the mitigation of large emissions associated with the counterfactual. In comparison within the scenarios where injection spreading systems were utilised in the counterfactual (a comparatively lower impact waste management practice), the overall scenario GHG intensities are greater - as a good practice process has been mitigated in the counterfactual resulting in less overall GHG benefit to the scenario.

A key theme that can be derived from analysing the results for the UK waste resources in Figure 3 is that low or even negative heat GHG intensity values can be achieved, particularly through the mitigation of high emission practices in the counterfactual, rather than the choice of specific bioenergy pathway. The GHG impact of 
activities and processes within the assumed counterfactual is therefore shown to be highly influential in determining the overall GHG performance of scenario, and thus influential in generating variability in GHG performance.

\subsubsection{GHG Performance of Generating Bioheat from Energy Crop Resources}

Figure 4: GHG Performance of Selected UK Willow SRC Energy Crop Bioenergy Scenarios - Reflecting Continual Energy Crop Production over a 40 Year Timeframe

Table 4: Key to Analyses Presented in Figure 4

The selected willow SRC bioenergy scenario GHG intensity values presented in Figure 4 also demonstrate high levels of variability. The analysis highlights that heat generated from willow SRC feedstocks produced on UK land may have GHG intensities that range from meeting the UK's sustainability target to exceeding the equivalent for natural gas generation.

Analysis of Figure 4 provides insight into the influences of this variability of heat GHG intensities across the scenarios. The influence of the scenario's assumed counterfactual pathway is clearly evident. Within the willow SRC scenarios where the analysed counterfactual pathway assumes that, 'the utilised land would otherwise have continued within its previous land use', all scenarios are shown to generate heat bioenergy with GHG intensities below that of natural gas, and depending on the characteristics of the bioenergy pathway the GHG intensities will meet the UK's sustainability target. In contrast, within the willow SRC scenarios where the analysed counterfactual pathway assumes that, 'the land would otherwise have reverted to a natural (forest) system', all the scenarios GHG intensities values are higher and may exceed the equivalent value for natural gas generation. Locking up carbon from the air to the land as the ecosystems continue to develop is shown to highly outweigh any benefits that may be gained through utilising the land to produce feedstock for bioenergy pathways - despite this option would not providing any productive income for land owners, any energy or other product. This highlights the importance of considering all potential counterfactuals when analysing the GHG performance of bioenergy pathways.

The GHG intensity values presented in Figure 4 demonstrate that there is only marginal differences in overall GHG performance through utilising the different forms of land considered in this study (abandoned arable/abandoned pasture/natural grassland), although other supply chain and bioenergy pathway life cycle characteristics can be highly influential on the overall GHG performance of the scenarios. For example, the use of SRC chip feedstocks that have been dried with no active energy (air dried) are shown to result in heat bioenergy with universally lower GHG intensities compared to the pellet feedstock scenarios where active energy is required to produce the feedstock. Further trends documented within Figure 4 are that the SRC scenarios that utilised large scale bioenergy conversion systems generate heat with marginally improved GHG intensities compared to scenarios where smaller scale systems are used - a consequence of the greater potential conversion efficiencies reflective of large scale bioenergy technologies.

\subsection{Analysing the Breakdown of Emissions and the Influence on Scenario GHG Performance}

Figure 5 and Figure 6 supported by the key in Table 5 provide a breakdown of the GHG emissions attributed to different processes and activities with both the counterfactual and bioenergy pathways. Figure 5 provides a breakdown of emissions within the food waste and cattle slurry scenarios (presented in Figure 3). Figure 6 provides a breakdown of emissions within the willow SRC scenarios (presented in Figure 4). This analysis may 
be used to evaluate how the overall GHG emissions from a given scenario may be attributed to specific activities and processes applicable to the bioenergy pathway.

Figure 5: Breakdown of the GHG Emissions per Tonne of Waste, as Proportional to Activities and Processes within both the Assumed Counterfactual and Bioenergy Pathways of the UK Food Waste \& Cattle Slurry Scenario Analyses

Figure 6: Breakdown of the GHG Emissions per Hectare of Land, as Proportional to Activities and Processes within both the Assumed Counterfactual and Bioenergy Pathways of the UK Willow SRC Scenario Analyses Reflecting Accumulated Emissions over a 40 Year Timeframe

Table 5: Key to Analyses Presented in Figure 5 and Figure 6

The counterfactual scenarios analysis presented in Figure 5 and Figure 6 provides a breakdown of the GHG emissions attributed to different activities and processes of the counterfactual pathways analysed within the respective slurry waste, food waste and willow SRC scenarios. It is these GHG emissions that may be avoided if the biomass resource / land is alternatively utilised within a bioenergy pathway. The bioenergy scenarios analysis presented in Figure 5 and Figure 6 provides a breakdown of the GHG emissions attributed to different activities and processes within the bioenergy pathways. These GHG emissions are directly emitted as a result the utilising the resource / land to ultimately generate bioenergy.

\subsubsection{The Slurry \& Food Waste Scenarios - Breakdown of Counterfactual Scenario GHG Emissions}

The analysis in Figure 5 highlights that the storage (SE-10) of agricultural wastes within lagoon storage systems is an agricultural practice with significant GHG impact. Fewer emissions are shown to result from the use of open tank systems where natural crusts are allowed to form, or within closed storage systems where the scenarios assume that generated GHG emissions are prevented through capture. Where lagoon systems are not utilised, the next greatest proportion of emissions are shown to be attributed to the spreading of the wastes on the land (SE-6), followed by emissions generated through the expenditure of energy / fuel to drive agricultural processes (SE-11). A further key trend highlighted within the analysis is that higher emissions are shown to result from scenarios where spreading of wastes is undertaken using broadcast systems, compared to scenarios where soil injection systems are utilised.

The analysis also provides insight into the scales of emissions potentially generated from the alternative food waste counterfactual pathways (SE-4). Sending food wastes to landfill sites is shown to result in the potential for large GHG emissions (mainly from methane, although these emissions are increasingly being captured by landfill gas installations on many UK sites). These emissions are shown to be potentially reduced by over $50 \%$ where the food wastes are alternatively managed through composting pathways.

\subsubsection{The Slurry \& Food Waste Scenarios - Breakdown of Bioenergy Scenario GHG Emissions}

The results ranges analysed within Figure 5 demonstrate that across the scenarios, emissions from two process / activity categories contribute the largest proportion of emissions: emissions following the spreading of digestate on the land (SE-6); and emissions attributed to agricultural processes (SE-11).

Emissions associated with processes attributed to bioenergy generation (SE-2) are reflective of the scenarios where biogas has been upgraded for potential injection to the grid. Where present the scale of these emissions is shown to be relatively small in proportion to the overall levels of emissions generated by the different processes throughout the scenarios. 


\subsubsection{The Willow SRC Scenarios - Breakdown of Counterfactual Scenario GHG Emissions}

The analysis within Figure 6 demonstrates that the key categories of emissions within the willow SRC counterfactual scenarios are shown to be natural land emissions (SE-6) from different categories of land, and the emissions flux between the atmosphere and land associated with the development of natural ecosystems (SE-7). The emissions profile of the two developed counterfactual scenarios demonstrate great contrast, where within the counterfactual pathway where, 'the land would revert to a natural (forest) system', the potential uptake of carbon from the atmosphere is highly significant. Thus, when this second counterfactual is assumed in the context of any scenario focusing on producing energy crops, mitigating this potential uptake of carbon from the atmosphere will adversely impact the GHG intensity of heat energy generated

\subsubsection{The Willow SRC Scenarios - Breakdown of Bioenergy Scenario GHG Emissions}

The result ranges analysed within Figure 6 are shown to be potentially large, highlighting that specific life cycle processes attributed to the different scenarios are highly influential in determining the overall GHG impact of a given scenario. The overall emissions associated with the scenarios where willow SRC pellets are produced, exceed the overall emissions of the scenarios where chips are the utilised feedstock. The main contrasts in the emission profiles associated with using the different forms of feedstock are shown to be attributed to the additional energy intensive processes required to produce pellets (SE-3). In contrast, the GHG emissions associated with transport processes (SE-9) are shown to be greater within the scenarios where energy crop chips are utilised - reflecting the greater bulk density and subsequent improved transport dynamics reflective of pellets compared to chips.

\subsubsection{Acknowledging the Methodology's Limitations and Uncertainties}

The scope of the research's analysis is reflective of the analysis boundaries (Figure 2) and the generated results are therefore a reflection of the research's specific resource and bioenergy scenarios. Processes with potential GHG impact outside of these analysis boundaries are not analysed and are therefore outside the scope of the research, and thus may represent potential limitations. As a result the research may provide further scientific evidence reflecting the GHG impact and bioenergy performances of pathways reflective of the developed scenarios, but are limited in their ability to analyse impacts outside of the analysis boundaries or to reflect wider macro-level GHG or consequential impacts if they were to be scaled up.

All styles of LCA analyses have inherent limitations when complex processes such as those analysed in the research are being assessed - these limitations largely resulting from the uncertainties of the specific modelling assumptions and input parameters. For example, the use of 'average' or 'mean' values to reflect key parameters will represent a key limitation for any analysis. There are many data assumptions and average values applied within a number of the research's modelling calculations. These are used to reflect wide literature consensus (such as, UK productivity yields for different crops / plants); and also where highly variable processes are modelled and literature highlighted ranges of likely values (such as, the likely $\mathrm{N}_{2} \mathrm{O}$ emissions from the spreading of different resources on land). There will also be certain levels of natural variability when modelling natural systems; this 'aleatory uncertainty' is largely irreducible to models (Hutton et al., 2010).

The analysis of the potential GHG impact associated with ILUC reflects a specific analysis theme with high uncertainty. As it is often impossible to determine whether ILUC is occurring and the extent that any impact can 
apportioned to specific events. Also if it is occurring, the specific types and nature of land use change taking place is unlikely to be known.

\section{4) Discussion}

The following section provides discussion of further themes and highlights some of the key trends derived from the research results.

\subsection{The Potential for Generating Low Carbon Heat from UK Biomass Resources}

By undertaking LCA analyses, this research found that heat with a GHG intensity less than the UK sustainability standard of $125.3 \mathrm{kgCO}_{2}{ }^{\text {eqv. }} / \mathrm{MWh}$ heat can be generated from each of the categories of UK biomass analysed: agricultural and food wastes; agricultural residues; and energy crops grown on UK land. However, a broad range of heat GHG intensities were calculated (Table 2). Heat GHG intensity values were found to both meet and in some cases fall well below the UK's threshold for sustainable heat, but also for some scenarios the GHG performances exceeded the equivalent heat GHG intensity value for heat generation from natural gas.

The high levels of GHG performance variability across the scenarios (as depicted in Figure 3 and Figure 4) were explained through assessing the emission profiles of selected scenarios and providing a breakdown of emissions (Figure 5 and Figure 6) as attributed to different activities and process inherent to each scenario. The inclusion of high GHG impact life cycle steps within the bioenergy scenario or the mitigation of GHG emissions from the assumed counterfactual were found to be highly influential in determining whether a particular bioenergy scenario would generate low carbon heat. If bioenergy pathways are to be incentivised and promoted based on their credentials as sources of 'low carbon renewable energy', greater depth has to be applied in describing the full life cycle and what counterfactuals they displace. Policies should focus on promoting best practice that avoids the most energy intensive steps (e.g. pelleting) where possible and should reward low carbon emissions along the supply chain rather than simply focusing on the resource / technology.

\subsection{The Influence of the Counterfactual on GHG Performance of Bioenergy Pathways}

The development and inclusion of counterfactual scenarios within bioenergy GHG balance analyses is a highly important theme. The European Environment Agency (EEA and European Environment Agency, 2007) concluded that the non-inclusion of counterfactuals when analysing the GHG impact of bioenergy pathways represents a basic but fundamental assumption error, as failure to account for the production or mitigation of GHG emissions within a counterfactual can result in a bioenergy scenario being wrongly identified as potentially saving or resulting in GHG emissions. At the same time the specific design of counterfactual scenarios can be subjective and thus highly contentious, as in the vast majority of cases it is difficult to 'know' what the real counterfactual would have been, and even harder to quantity any consequential LCA emissions that may / may not have resulted from that counterfactual (Adams et al., 2013). As such, it is better to include counterfactuals within any bioenergy GHG balance analyses, and be aware that their inclusion affects the interpretation of any outputs.

The influence of counterfactuals on the GHG performance of the bioenergy scenarios are evidenced across all the bioenergy scenarios evaluated in this research. One of the most striking examples is shown within waste bioenergy scenarios (Figure 3 and Figure 5); where the mitigation of high GHG impacting agricultural processes 
such as storing slurries within lagoons, or disposing of food wastes within landfill, was shown to result in large potential GHG savings where the resource was instead utilised for bioenergy generation. Alternatively, where the assumed counterfactuals resulted in the mitigation of low GHG impacting processes such as closed tank slurry storage - the resulting GHG savings associated with using the resource for bioenergy generation were much diminished.

GHG emissions savings that have been prevented through the mitigation of a high impact activity within the counterfactual will likely be attributed to the emission inventories of sectors other than the energy sector. For example, reducing large emissions through avoiding spreading slurry on the land or diverting food waste to landfill will reflect emission savings for agricultural and waste management emission inventories respectively.

\subsection{Lessons and Implications for Policy}

A key implication of the research findings for policy makers stems from the conclusions that: variations in the GHG performance for any given biomass resource are found to reflect the embodied GHG impacts of the life cycle steps and processes specific to the resource and bioenergy pathways. It is therefore not possible to accurately benchmark categories of biomass resource in terms of their potential GHG performance, but it is possible to identify specific life cycle processes and activities inherent to biomass resources that may either enhance or reduce the GHG performance of a given bioenergy generated pathways. Therefore policies and sophisticated bioenergy GHG impact assessment methodologies should be developed that evaluate the whole life cycle and specific steps of biomass resources and bioenergy pathways. Policies should promote and incentivise the: use of specific biomass resources that will result in the mitigation of high GHG impact activities; and use of bioenergy pre-treatment and conversion technologies that will deliver low carbon bioenergy.

The potential wider emission benefits associated with selected bioenergy pathways within the research represents an attractive characteristic of bioenergy and opportunity for generating wider emissions savings. As such, policy focus and incentive mechanisms should be developed that take into consideration the synergies and consequences of different bioenergy pathways, moving away from the focus of bioenergy 'maximise renewable generation' rather than to simply 'reduce GHG emissions' (Thornley, 2016). Table 6 provides a summary of key lessons and recommendations for policy to enhance the GHG performance of heat bioenergy generated from biomass resources.

Table 6: Key Lessons for Increasing the GHG Performance of Bioenergy Pathways

\section{5) Conclusions}

This research analysed the potential GHG performance of utilising UK biomass resources to generate heat bioenergy. LCA analyses were undertaken to evaluate a series of developed bioenergy scenarios that focused on utilising: agricultural wastes and food wastes through anaerobic digestion pathways; straw agricultural residues and UK grown energy crops through combustion pathways.

The research found that the potential heat bioenergy generated from the analysed scenarios reflected a broad range of GHG performances - some heat GHG intensity values were found to meet and in some cases fall well below the UK's threshold for sustainable heat. The majority of scenarios demonstrated potential GHG savings 
compared to conventional fossil fuel generation, although selected scenarios highlighted potential greater GHG impact.

The variations in GHG performance for any given biomass resource were found to reflect the embodied GHG impacts of the life cycle steps and processes specific to the resource, bioenergy pathways and assumed counterfactuals. Thus it is not possible to accurately benchmark categories of biomass resource or bioenergy systems in terms of their potential GHG performance. However it is possible to identify specific life cycle processes and activities inherent to biomass resources that may either enhance or reduce the GHG performance of a given bioenergy generated pathways.

The characteristics of the analysed counterfactual scenarios were found to potentially control high influence on the GHG performance. The mitigation of high GHG impacting processes within assumed counterfactual scenarios was shown to result in large potential GHG savings where the resources are instead utilised for bioenergy generation. Alternatively, where the assumed counterfactuals resulted in the mitigation of reflectively low GHG impacting processes, or GHG absorbing processes (e.g. regeneration of abandoned land to forest), the resulting GHG savings associated with using the resource for bioenergy generation are shown to be much diminished.

Policies and bioenergy GHG impact assessments should focus on the whole life cycle and specific steps of bioenergy pathways in order to promote and incentivise: the use of specific biomass resources that will result in the mitigation of high GHG impact activities; and use of bioenergy pre-treatment and conversion technologies that will deliver low carbon bioenergy. 


\section{6) References}

Adams, P., Bows, A., Gilbert, P., Hammond, J., Howard, D., Lee, R., McNamara, N., Thornley, P., Whittaker, C., Whitaker, J., 2013. Understanding Greenhouse Gas Balances of Bioenergy Systems. Manchester.

Ahlgren, S., Di Lucia, L., 2014. Indirect Land Use Changes of Biofuel Production - a Review of Modelling Efforts and Policy Developments in the European Union. Biotechnol. Biofuels 7, 35.

Bowyer, C., Baldock, D., Kretschmer, B., Polakova, J., 2012. The GHG Emissions Intensity of Bioenergy: Does Bioenergy have a Role to Play in Reducing GHG Emissions of Europe's Economy? London.

DECC, 2014a. BEAC (Biomass Emission and Counterfactual) Model.

DECC, 2014b. Personal Conversations with the DECC Renewable Heat Incentive Team.

DECC, 2013. UK Renewable Energy Roadmap Update 2013. London.

DECC, 2009. The UK Renewable Energy Strategy.

EEA, 2007. Estimating the Environmentally Compatible Bioenergy Potential from Agriculture. Copenhagen.

European Commission, 2010. Report on Sustainability Requirements for the Use of Solid and Gaseous Biomass Sources in Electricity, Heating and Cooling. Brussels.

European Commission, 2009. The Promotion of the Use of Energy from Renewable Sources. Brussels.

Haberl, H., Sprinz, D., Bonazountas, M., Cocco, P., Desaubies, Y., Henze, M., Hertel, O., Johnson, R.K., Kastrup, U., Laconte, P., Lange, E., Novak, P., Paavola, J., Reenberg, A., van den Hove, S., Vermeire, T., Wadhams, P., Searchinger, T., 2012. Correcting a fundamental error in greenhouse gas accounting related to bioenergy. Energy Policy 45-222, 18-23. doi:10.1016/j.enpol.2012.02.051

Hutton, C., Vamvakeridou-Lyroudi, L., Kapelan, Z., Savic, D., 2010. Real-Time Modelling and Data Assimilation Techniques for Improving the Accuracy of Model Predictions: Scientific Report. Brussels.

MacKay, D., Stephenson, A., 2014. Life Cycle Impacts of Biomass Electricity in 2020. London.

Nguyen, T.L.T., Gheewala, S.H., Sagisaka, M., 2010. Greenhouse gas savings potential of sugar cane bioenergy systems. J. Clean. Prod. 18, 412-418. doi:10.1016/j.jclepro.2009.12.012

Panoutsou, C., Elbersen, B., Joost van Stralen, J., Uslu, A., Fritsche, U., 2010. The Role of Straw for Heat \& Electricity in EU27 Member States in 2020 and in 2030 with Respect to Costs and Sustainability Criteria. London.

Röder, M., Whittaker, C., Thornley, P., 2015. How certain are greenhouse gas reductions from bioenergy? Life cycle assessment and uncertainty analysis of wood pellet-to-electricity supply chains from forest residues. Biomass and Bioenergy 79, 50-63. doi:10.1016/j.biombioe.2015.03.030

Thornley, P., 2016. The Role of Policy Regimes in Maximizing GHG Savings in Bioenergy Systems. TBC TBC, TBC.

Thornley, P., Gilbert, P., Shackley, S., Hammond, J., 2015. Maximizing the greenhouse gas reductions from biomass: The role of life cycle assessment. Biomass and Bioenergy 81, 35-43. doi:10.1016/j.biombioe.2015.05.002

UK Government, 2008. UK Climate Change Act. United Kingdom.

United Nations, 1998. Kyoto Protocol to the United Nations Framework Convention on Climate Change. http://unfccc.int/resource/docs/convkp/kpeng.pdf

Upham, P., Tomei, J., 2010. Biofuels \& Regulatory Co-Production: Critical Stakeholder Perceptions of Carbon and Sustainability Reporting in the UK Renewable Transport Fuel Obligation.

Welfle, A., 2014. Biomass Resource Analyses \& Future Bioenergy Scenarios. University of Manchester.

Welfle, A., Gilbert, P., Thornley, P., 2014a. Securing a Bioenergy Future without Imports. Energy Policy 68, 114. doi:10.1016/j.enpol.2013.11.079

Welfle, A., Gilbert, P., Thornley, P., 2014b. Increasing Biomass Resource Availability through Supply Chain Analysis. Biomass Bioenergy 70, 249-266. doi:10.1016/j.biombioe.2014.08.001 